International Journal of Pure and Applied Mathematics

Volume 90 No. 3 2014, 371-385

ISSN: 1311-8080 (printed version); ISSN: 1314-3395 (on-line version)

url: http://www.ijpam.eu

doi: http://dx.doi.org/10.12732/ijpam.v90i3.10

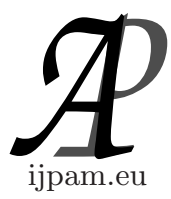

\title{
MINIMUM COVERING HARARY ENERGY OF A GRAPH
}

\author{
M.R. Rajesh Kanna ${ }^{1}$, B.N. Dharmendra ${ }^{2}$, R. Pradeep Kumar ${ }^{3}$ \\ ${ }^{1,2}$ Post Graduate Department of Mathematics \\ Maharani's Science College for Women \\ J.L.B. Road, Mysore, 570 005, INDIA \\ ${ }^{3}$ Research and Development Centre \\ Bharathiar University \\ Coimbatore, 641 046, INDIA
}

\begin{abstract}
In this paper we computed minimum covering Harary energies of a star graph, complete graph, crown graph, bipartite graph and cocktail party graphs. Upper and lower bounds for minimum covering Harary energies are also established.
\end{abstract}

AMS Subject Classification: 05C50, 05C69

Key Words: minimum covering set, minimum covering Harary matrix, minimum covering Harary eigenvalues, minimum covering Harary energy of a graph

\section{Introduction}

The concept of energy of a graph was introduced by I. Gutman [11] in the year 1978. Let $G$ be a graph with $n$ vertices $\left\{v_{1}, v_{2}, \ldots, v_{n}\right\}$ and $m$ edges. Let $A=\left(a_{i j}\right)$ be the adjacency matrix of the graph. The eigenvalues $\lambda_{1}, \lambda_{2}, \cdots, \lambda_{n}$

Received: October 22, 2013

(c) 2014 Academic Publications, Ltd. url: www.acadpubl.eu

${ }^{\S}$ Correspondence author 
of $A$, assumed in non increasing order, are the eigenvalues of the graph $G$. As $A$ is real symmetric, the eigenvalues of $G$ are real with sum equal to zero. The energy $E(G)$ of $G$ is defined to be the sum of the absolute values of the eigenvalues of $G$. i.e., $E(G)=\sum_{i=1}^{n}\left|\lambda_{i}\right|$.

For details on the mathematical aspects of the theory of graph energy see the reviews [12], papers $[6,7,13]$ and the references cited there in. The basic properties including various upper and lower bounds for energy of a graph have been established in $[15,17]$, and it has found remarkable chemical applications in the molecular orbital theory of conjugated molecules $[9,14]$.

The Harary matrix of $G$ is the square matrix of order $n$ whose $(i, j)$-entry is $\frac{1}{d_{i j}}$ where $d_{i j}$ is the distance between the vertices $v_{i}$ and $v_{j}$. Let $\rho_{1}, \rho_{2}, \ldots, \rho_{n}$ be the eigenvalues of the Harary matrix of $G$. The Harary energy $H E$ is defined by $H E=H E(G):=\sum_{i=1}^{n}\left|\rho_{i}\right|$. Detailed studies on distance energy and Harary energy can be found in $[5,10,21,23,8,22]$.

Recently Prof. Chandrashekar Adiga et al. [1] have defined the minimum covering energy, $E_{C}(G)$ of a graph $G$ which depends on its particular minimum cover $C$. Motivated by this paper, we introduced the concept of minimum covering Harary energy $\mathrm{HE}_{C}(G)$ of a graph $G$ and computed minimum covering Harary energies of a star graph, complete graph, crown graph, bipartite graph and cocktail party graphs. Upper and lower bounds for $H E_{C}(G)$ are also established.

Further, studies on minimum covering energy, maximum degree energy, minimum dominating energy, minimum covering distance energies can be found in $[1,2,18,19,20]$ and the references cited there in.

\section{Definitions and Example}

\subsection{The Minimum Covering Energy of a Graph}

Let $G$ be a simple graph of order $n$ with vertex set $V=\left\{v_{1}, v_{2}, \ldots, v_{n}\right\}$ and edge set $E$. A subset $C$ of $V$ is called a covering set of $G$ if every edge of $G$ is incident to at least one vertex of $C$. Any covering set with minimum cardinality is called a minimum covering set. Let $C$ be a minimum covering set of a graph $G$. The minimum covering matrix of $G$ is the $n \times n$ matrix defined by $A_{C}(G):=\left(a_{i j}\right)$, 
where $a_{i j}= \begin{cases}1 & \text { if } v_{i} v_{j} \in E \\ 1 & \text { if } i=j \text { and } v_{i} \in C \\ 0 & \text { otherwise }\end{cases}$

The characteristic polynomial of $A_{C}(G)$ is denoted by $f_{n}(G, \lambda)=\operatorname{det}(\lambda I-$ $A_{C}(G)$ ). The minimum covering eigenvalues of the graph $G$ are the eigenvalues of $A_{C}(G)$. Since $A_{C}(G)$ is real and symmetric, its eigenvalues are real numbers and we label them in non-increasing order $\lambda_{1} \geqslant \lambda_{2} \geqslant \cdots \geqslant \lambda_{n}$. The minimum covering energy of $G$ is then defined as $E_{C}(G)=\sum_{i=1}^{n}\left|\lambda_{i}\right|$.

\subsection{The Minimum Covering Harary Energy of a Graph}

Let $G$ be a simple graph of order $n$ with vertex set $V=\left\{v_{1}, v_{2}, \ldots, v_{n}\right\}$ and edge set $E$. Let $C$ be a minimum covering set of a graph $G$. The minimum covering Harary matrix of $G$ is the $n \times n$ matrix defined by $H_{C}(G):=\left(h_{i j}\right)$, where $h_{i j}= \begin{cases}1 & \text { if } i=j \text { and } v_{i} \in C \\ 0 & \text { if } i=j \text { and } v_{i} \notin C \\ \frac{1}{d\left(v_{i}, v_{j}\right)} & \text { otherwise }\end{cases}$

The characteristic polynomial of $H_{C}(G)$ is denoted by $f_{n}(G, \rho)=\operatorname{det}(\rho I-$ $H_{C}(G)$ ). The minimum covering Harary eigenvalues of the graph $G$ are the eigenvalues of $H_{C}(G)$. Since $H_{C}(G)$ is real and symmetric, its eigenvalues are real numbers and we label them in non-increasing order $\rho_{1} \geqslant \rho_{2} \geqslant \cdots \geqslant \rho_{n}$. The minimum covering Harary energy of $G$ is defined as $H E_{C}(G):=\sum_{i=1}^{n}\left|\rho_{i}\right|$ Note that the trace of $H_{C}(G)=|C|$.

In this paper, we are interested in studying mathematical aspects of the minimum covering Harary energy of a graph. The application of minimum covering Harary energy in other branches of science have to be investigated.

Example 1. The possible minimum covering sets for the following graph $G$ (see Figure 1) are i) $C_{1}=\left\{v_{1}, v_{2}, v_{5}\right\}$, ii) $C_{2}=\left\{v_{2}, v_{4}, v_{5}\right\}$, iii) $C_{3}=\left\{v_{1}, v_{3}, v_{5}\right\}$. 


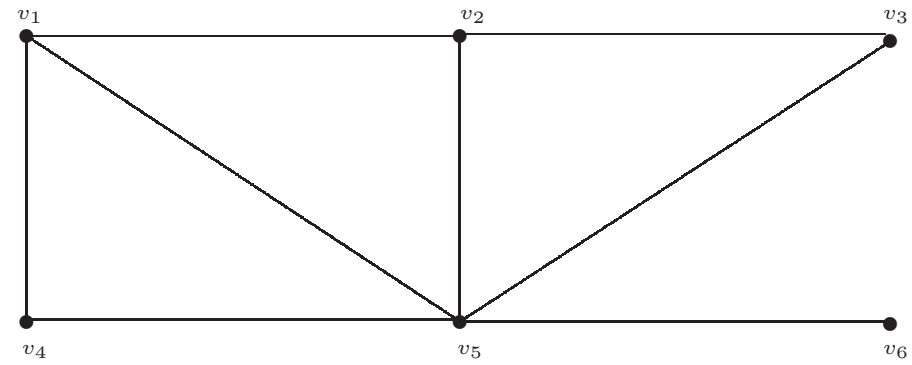

Figure 1

i) $H_{C_{1}}(G)=\left(\begin{array}{cccccc}1 & 1 & 1 / 2 & 1 & 1 & 1 / 2 \\ 1 & 1 & 1 & 1 / 2 & 1 & 1 / 2 \\ 1 / 2 & 1 & 0 & 1 / 2 & 1 & 1 / 2 \\ 1 & 1 / 2 & 1 / 2 & 0 & 1 & 1 / 2 \\ 1 & 1 & 1 & 1 & 1 & 1 \\ 1 / 2 & 1 / 2 & 1 / 2 & 1 / 2 & 1 & 0\end{array}\right)$

Characteristic equation is $\rho^{6}-3 \rho^{5}-\frac{27}{4} \rho^{4}-2 \rho^{3}+\frac{23}{16} \rho^{2}+\frac{3}{8} \rho-\frac{1}{8}=0$.

Minimum covering Harary eigenvalues are $\rho_{1} \approx .3090169944, \rho_{2} \approx-.8090169944$, $\rho_{3} \approx .2577609956, \rho_{4} \approx 4.560355576, \rho_{5} \approx-.5641882616, \rho_{6} \approx-.7539283099$.

Minimum covering Harary energy, $H E_{C_{1}}(G) \approx 7.254267133$.

ii) $H_{C_{2}}(G)=\left(\begin{array}{cccccc}0 & 1 & 1 / 2 & 1 & 1 & 1 / 2 \\ 1 & 1 & 1 & 1 / 2 & 1 & 1 / 2 \\ 1 / 2 & 1 & 0 & 1 / 2 & 1 & 1 / 2 \\ 1 & 1 / 2 & 1 / 2 & 1 & 1 & 1 / 2 \\ 1 & 1 & 1 & 1 & 1 & 1 \\ 1 / 2 & 1 / 2 & 1 / 2 & 1 / 2 & 1 & 0\end{array}\right)$

Characteristic equation is $\rho^{6}-3 \rho^{5}-\frac{27}{4} \rho^{4}-\frac{5}{4} \rho^{3}+\frac{39}{16} \rho^{2}+\frac{13}{16} \rho-\frac{1}{16}=0$.

Minimum covering Harary eigenvalues are $\rho_{1} \approx .6486997113, \rho_{2} \approx .593985$ $3888, \rho_{3} \approx 4.524661925, \rho_{4} \approx-.5693526951, \rho_{5} \approx-.6596347450, \rho_{6} \approx-.954529$ 8444.

Minimum covering Harary energy, $H E_{C_{2}}(G) \approx 7.950864309$.

$\therefore$ Minimum covering Harary energy depends on the covering set.

\section{Minimum Covering Harary Energy of some Standard Graphs}

Definition 3.1. The Cocktail party graph is denoted by $K_{n \times 2}$, is a graph having the vertex set $V=\bigcup_{i=1}^{n}\left\{u_{i}, v_{i}\right\}$ and the edge set $E=\left\{u_{i} u_{j}, v_{i} v_{j}: i \neq\right.$ $j\} \bigcup\left\{u_{i} v_{j}, v_{i} u_{j}: 1 \leq i<j \leq n\right\}$. 
Theorem 3.1. The minimum covering Harary energy of Cocktail party graph $K_{n \times 2}$ is $(n-1)+\sqrt{4 n^{2}+4 n-7}$.

Proof. Let $K_{n \times 2}$ be the Cocktail party graph with vertex set $V=\bigcup_{i=1}^{n}\left\{u_{i}, v_{i}\right\}$. The minimum covering set is $C=\bigcup^{n-1}\left\{u_{i}, v_{i}\right\}$.

$$
\text { Then } H_{C}\left(K_{n \times 2}\right)=\left(\begin{array}{ccccccccc}
1 & 1 / 2 & 1 & 1 & \ldots & 1 & 1 & 1 & 1 \\
1 / 2 & 1 & 1 & 1 & \ldots & 1 & 1 & 1 & 1 \\
1 & 1 & 1 & 1 / 2 & \ldots & 1 & 1 & 1 & 1 \\
1 & 1 & 1 / 2 & 1 & \ldots & 1 & 1 & 1 & 1 \\
\vdots & \vdots & \vdots & \vdots & \ddots & \vdots & \vdots & \vdots & \vdots \\
1 & 1 & 1 & 1 & \ldots & 1 & 1 / 2 & 1 & 1 \\
1 & 1 & 1 & 1 & \ldots & 1 / 2 & 1 & 1 & 1 \\
1 & 1 & 1 & 1 & \ldots & 1 & 1 & 0 & 1 / 2 \\
1 & 1 & 1 & 1 & \ldots & 1 & 1 & 1 / 2 & 0
\end{array}\right)
$$

Characteristic equation is

$$
\frac{1}{4^{n}}(2 \rho+1)^{n-1}(2 \rho-1)^{n-1}\left[4 \rho^{2}-8(n-1) \rho-(12 n-11)\right]=0
$$

Minimum covering Harary eigenvalues are $\rho=\frac{-1}{2}[(n-1)$ times $], \rho=\frac{1}{2}[(n-1)$ times], $\rho=\frac{(2 n-2) \pm \sqrt{4 n^{2}+4 n-7}}{2}$ [one time each]

Minimum covering Harary energy, $H E_{C}\left(K_{n \times 2}\right)$

$$
\begin{aligned}
& =\left|\frac{-1}{2}\right|(n-1)+\left|\frac{1}{2}\right|(n-1)+\left|\frac{(2 n-2)+\sqrt{4 n^{2}+4 n-7}}{2}\right|+ \\
& =\left|\frac{(2 n-2)-\sqrt{4 n^{2}+4 n-7}}{2}\right| \\
& =(n-1)+\sqrt{4 n^{2}+4 n-7} .
\end{aligned}
$$

Theorem 3.2. For $n \geq 3$, the minimum covering Harary energy of Star graph $K_{1, n-1}$ is equal to $\frac{(n+1)-\sqrt{n^{2}+10 n+9}}{2}$.

Proof. Consider the Star graph $K_{1, n-1}$ with vertex set $V=\left\{v_{0}, v_{1}, v_{2}, \ldots, v_{n-1}\right\}$. The Minimum covering set $C=\left\{v_{0}\right\}$. Then 


$$
H_{C}\left(K_{1, n-1}\right)=\left(\begin{array}{ccccc}
1 & 1 & 1 & \ldots & 1 \\
1 & 0 & \frac{1}{2} & \ldots & \frac{1}{2} \\
1 & \frac{1}{2} & 0 & \ldots & \frac{1}{2} \\
\vdots & \vdots & \vdots & \ddots & \vdots \\
1 & \frac{1}{2} & \frac{1}{2} & \ldots & 0
\end{array}\right)_{n \times n}
$$

Characteristic equation is $\frac{1}{2^{n}}(2 \rho+1)^{n-1}\left[2 \rho^{2}-(n+1) \rho-(n-1)\right]=0$

The minimum covering Harary eigenvalues are each].

$$
\rho=\frac{-1}{2}[(n-1) \text { times }], \quad \rho=\frac{(n+1) \pm \sqrt{n^{2}+10 n+9}}{4} \text { [one time }
$$

Minimum covering Harary energy is, $H E_{C}\left(K_{1, n-1}\right)$

$$
\begin{aligned}
& =\left|\frac{-1}{2}\right|(n-1)+\left|\frac{(n+1)+\sqrt{n^{2}+10 n+9}}{4}\right|+\left|\frac{(n+1)-\sqrt{n^{2}+10 n+9}}{4}\right| \\
& =\frac{(n+1)-\sqrt{n^{2}+10 n+9}}{2} .
\end{aligned}
$$

Definition 3.2. The Crown graph $S_{n}^{0}$ for an integer $n \geq 2$ is the graph with vertex set $\left\{u_{1}, u_{2}, \ldots, u_{n}, v_{1}, v_{2}, \ldots, v_{n}\right\}$ and edge set $\left\{u_{i} v_{j}: 1 \leq i, j \leq n, i \neq\right.$ $j\} . \quad \therefore S_{n}^{0}$ coincides with the Complete bipartite graph $K_{n, n}$ with horizontal edges removed.

Theorem 3.3. The minimum covering Harary energy of the Crown graph $S_{n}^{0}$ is equal to $\frac{(5 n-5)+\sqrt{36 n^{2}-48 n+25}}{3}$

Proof. For the Crown graph $S_{n}^{0}$ with vertex set $V=\left\{u_{1}, u_{2}, \ldots, u_{n}, v_{1}, v_{2}, \ldots, v_{n}\right\}$. The minimum covering set is $C=\left\{u_{1}, u_{2}, \ldots, u_{n}\right\}$. Then 


$$
H_{C}\left(S_{n}^{0}\right)=\left(\begin{array}{cccccccccc}
1 & \frac{1}{2} & \frac{1}{2} & \ldots & \frac{1}{2} & \frac{1}{3} & 1 & 1 & \ldots & 1 \\
\frac{1}{2} & 1 & \frac{1}{2} & \ldots & \frac{1}{2} & 1 & \frac{1}{3} & 1 & \ldots & 1 \\
\frac{1}{2} & \frac{1}{2} & 1 & \ldots & \frac{1}{2} & 1 & 1 & \frac{1}{3} & \ldots & 1 \\
\vdots & \vdots & \vdots & \ddots & \vdots & \vdots & \vdots & \vdots & \ddots & \vdots \\
\frac{1}{2} & \frac{1}{2} & \frac{1}{2} & \ldots & 1 & 1 & 1 & 1 & \ldots & \frac{1}{3} \\
\frac{1}{3} & 1 & 1 & \ldots & 1 & 0 & \frac{1}{2} & \frac{1}{2} & \ldots & \frac{1}{2} \\
1 & \frac{1}{3} & 1 & \ldots & 1 & \frac{1}{2} & 0 & \frac{1}{2} & \ldots & \frac{1}{2} \\
1 & 1 & \frac{1}{3} & \ldots & 1 & \frac{1}{2} & \frac{1}{2} & 0 & \ldots & \frac{1}{2} \\
\vdots & \vdots & \vdots & \ddots & \vdots & \vdots & \vdots & \vdots & \ddots & \vdots \\
1 & 1 & 1 & \ldots & \frac{1}{3} & \frac{1}{2} & \frac{1}{2} & \frac{1}{2} & \ldots & 0
\end{array}\right)_{(2 n \times 2 n)}
$$

Characteristic equation is

$\frac{1}{36^{n}}(6 \rho-5)^{n-1}(6 \rho+5)^{n-1}\left[36 \rho^{2}-36 n \rho-\left(27 n^{2}--48 n+25\right)\right]=0$.

Minimum covering Harary eigenvalues are $\rho=\frac{5}{6}[(n-1)$ times $]$,

$\rho=\frac{-5}{6}[(n-1)$ times $], \rho=\frac{3 n \pm \sqrt{36 n^{2}-48 n+25}}{6}[1$ time each $]$

Minimum covering Harary energy, $H E_{C}\left(K_{n \times 2}\right)$

$$
\begin{aligned}
& =\left|\frac{5}{6}\right|(n-1)+\left|\frac{-5}{6}\right|(n-1)+\left|\frac{3 n+\sqrt{36 n^{2}-48 n+25}}{6}\right|+ \\
& =\frac{\left|\frac{3 n-\sqrt{36 n^{2}-48 n+25}}{6}\right|}{3} \\
& =\frac{(5 n-5)+\sqrt{36 n^{2}-48 n+25}}{3} .
\end{aligned}
$$

Theorem 3.4. The minimum covering Harary energy of a Complete bipartite graph $K_{m, n},(m \leq n)$ is

$$
\frac{(m+n)+\sqrt{\left(m^{2}+n^{2}+14 m n+4 m-4 n+4\right)}}{2} .
$$

Proof. For the Complete bipartite graph $K_{m, n}(m \leq n)$ with vertex set $V=\left\{u_{1}, u_{2}, \ldots, u_{m}, v_{1}, v_{2}, \ldots, v_{n}\right\}$ the minimum covering set is $C=\left\{u_{1}, u_{2}, \ldots, u_{m}\right\}$. Then 


$$
H_{C}\left(K_{m, n}\right)=\left(\begin{array}{cccccccccc}
1 & \frac{1}{2} & \frac{1}{2} & \ldots & \frac{1}{2} & 1 & 1 & 1 & \ldots & 1 \\
\frac{1}{2} & 1 & \frac{1}{2} & \ldots & \frac{1}{2} & 1 & 1 & 1 & \ldots & 1 \\
\frac{1}{2} & \frac{1}{2} & 1 & \ldots & \frac{1}{2} & 1 & 1 & 1 & \ldots & 1 \\
\vdots & \vdots & \vdots & \ddots & \vdots & \vdots & \vdots & \vdots & \ddots & \vdots \\
\frac{1}{2} & \frac{1}{2} & \frac{1}{2} & \ldots & 1 & 1 & 1 & 1 & \ldots & 1 \\
1 & 1 & 1 & \ldots & 1 & 0 & \frac{1}{2} & \frac{1}{2} & \ldots & \frac{1}{2} \\
1 & 1 & 1 & \ldots & 1 & \frac{1}{2} & 0 & \frac{1}{2} & \ldots & \frac{1}{2} \\
1 & 1 & 1 & \ldots & 1 & \frac{1}{2} & \frac{1}{2} & 0 & \ldots & \frac{1}{2} \\
\vdots & \vdots & \vdots & \ddots & \vdots & \vdots & \vdots & \vdots & \ddots & \vdots \\
1 & 1 & 1 & \ldots & 1 & \frac{1}{2} & \frac{1}{2} & \frac{1}{2} & \ldots & 0
\end{array}\right)_{(m+n) \times(m+n)}
$$

Characteristic equation is

$$
\frac{(2 \rho-1)^{m-1}(2 \rho+1)^{n-1}\left[4 \rho^{2}-2(m+n) \rho-(3 m n+m-n+1)\right]}{2^{m+n}}=0 .
$$

Minimum covering Harary eigenvalues are $\rho=\frac{1}{2}[(m-1)$ times $]$,

$\rho=\frac{-1}{2}[(n-1)$ times $]$ and $\rho=\frac{(m+n) \pm \sqrt{m^{2}+n^{2}+14 m n+4 m-4 n+4}}{4}$ [one time each].

Minimum covering Harary energy is, $H E_{C}\left(K_{m, n}\right)=$

$$
\begin{aligned}
& \left|\frac{1}{2}\right|(m-1)+\left|\frac{-1}{3}\right|(n-1)+\left|\frac{(m+n)+\sqrt{\left(m^{2}+n^{2}+14 m n+4 m-4 n+4\right)} \mid}{4}\right| \\
+ & \mid \frac{(m+n)-\sqrt{\left(m^{2}+n^{2}+14 m n+4 m-4 n+4\right)} \mid}{4} \\
= & \frac{(m+n)+\sqrt{\left(m^{2}+n^{2}+14 m n+4 m-4 n+4\right)}}{2}
\end{aligned}
$$

Theorem 3.5. For $n \geq 2$, the minimum covering Harary energy of Complete graph $K_{n}$ is $\sqrt{(n+3)(n-1)}$.

Proof. For complete graphs the minimum covering Harary matrix is same as minimum covering matrix [1], therefore the Minimum covering Harary energy is equal to Minimum covering energy. 


\section{Properties of Minimum Covering Harary Eigenvalues}

Theorem 4.1. Let $G$ be a simple graph with vertex set $V=\left\{v_{1}, v_{2}, \ldots, v_{n}\right\}$, edge set $E$ and $C=\left\{u_{1}, u_{2}, \ldots, u_{k}\right\}$ be a minimum covering set. If $\rho_{1}, \rho_{2}, \ldots, \rho_{n}$ are the eigenvalues of minimum covering Harary matrix $H_{C}(G)$ then (i) $\sum_{i=1}^{n} \rho_{i}$ $=|C|$

(ii) $\sum_{i=1}^{n} \rho_{i}^{2}=|C|+2 \sum_{i<j} \frac{1}{d\left(v_{i}, v_{j}\right)^{2}}$.

Proof. (i) We know that the sum of the eigenvalues of $H_{C}(G)$ is the trace of $H_{C}(G)$

$$
\therefore \sum_{i=1}^{n} \rho_{i}=\sum_{i=1}^{n} h_{i i}=|C| .
$$

(ii) Similarly the sum of squares of the eigenvalues of $H_{C}(G)$ is trace of $\left[H_{C}(G)\right]^{2}$

$$
\begin{aligned}
\therefore \sum_{i=1}^{n} \rho_{i}^{2} & =\sum_{i=1}^{n} \sum_{j=1}^{n} h_{i j} h_{j i} \\
& =\sum_{i=1}^{n}\left(h_{i i}\right)^{2}+\sum_{i \neq j} h_{i j} h_{j i} \\
& =\sum_{i=1}^{n}\left(h_{i i}\right)^{2}+2 \sum_{i<j}\left(h_{i j}\right)^{2} \\
& =|C|+2 \sum_{i<j} \frac{1}{d\left(v_{i}, v_{j}\right)^{2}}
\end{aligned}
$$

Corollary 4.2. Let $G$ be a $(n, m)$ simple graph with diameter 2 and $C=$ $\left\{u_{1}, u_{2}, \ldots, u_{k}\right\}$ be a minimum covering set. If $\rho_{1}, \rho_{2}, \ldots, \rho_{n}$ are the eigenvalues of minimum covering Harary matrix $H_{C}(G)$ then $\sum_{i=1}^{n} \rho_{i}^{2}=\frac{4|C|+n^{2}-n+6 m}{4}$

Proof. We know that in $H_{C}(G)$ there are $2 m$ elements with 1 and $n(n-1)-$ $2 m$ elements with $\frac{1}{2}$ and hence corollary follows from the above theorem. 


\section{Bounds for Minimum Covering Harary Energy}

Similar to McClelland's [17] bounds for energy of a graph, bounds for $H E_{C}(G)$ are given in the following theorem.

Theorem 5.1. Let $G$ be a simple $(n, m)$ graph. If $C$ is the minimum covering set and $P=\left|H_{C}(G)\right|$ then

$$
\begin{aligned}
& \sqrt{|C|+2 \sum_{i<j} \frac{1}{d\left(v_{i}, v_{j}\right)^{2}}+n(n-1) P^{\frac{2}{n}}} \\
& \quad \leq H E_{C}(G) \leq \sqrt{n\left(|C|+2 \sum_{i<j} \frac{1}{d\left(v_{i}, v_{j}\right)^{2}}\right)}
\end{aligned}
$$

where $|C|$ is the cardinality of minimum covering set.

Proof.

Cauchy Schwarz inequality is $\left(\sum_{i=1}^{n} a_{i} b_{i}\right)^{2} \leq\left(\sum_{i=1}^{n} a_{i}^{2}\right)\left(\sum_{i=1}^{n} b_{i}^{2}\right)$

$$
\text { If } a_{i}=1, b_{i}=\left|\rho_{i}\right| \text { then }\left(\sum_{i=1}^{n}\left|\rho_{i}\right|\right)^{2} \leq\left(\sum_{i=1}^{n} 1\right)\left(\sum_{i=1}^{n} \rho_{i}^{2}\right)
$$

By using Theorem (4.1), we obtain

$$
\begin{gathered}
{\left[H E_{C}(G)\right]^{2} \leq n\left(|C|+2 \sum_{i<j} \frac{1}{d\left(v_{i}, v_{j}\right)^{2}}\right)} \\
\Longrightarrow H E_{C}(G) \leq \sqrt{n\left(|C|+2 \sum_{i<j} \frac{1}{d\left(v_{i}, v_{j}\right)^{2}}\right)}
\end{gathered}
$$

Since arithmetic mean is not smaller than geometric mean we have

$$
\begin{aligned}
\frac{1}{n(n-1)} \sum_{i \neq j}\left|\rho_{i} \| \rho_{j}\right| & \geq\left[\prod_{i \neq j}\left|\rho_{i}\right|\left|\rho_{j}\right|\right] \frac{1}{\frac{n(n-1)}{n}} \\
& =\left[\prod_{i=1}^{n}\left|\rho_{i}\right|^{2(n-1)}\right] \frac{1}{n(n-1)} \\
& =\left[\prod_{i=1}^{n}\left|\rho_{i}\right|\right]^{\frac{2}{n}}
\end{aligned}
$$




$$
\begin{gathered}
=\left|\prod_{i=1}^{n} \rho_{i}\right|^{\frac{2}{n}} \\
=\left|H_{C}(G)\right|^{\frac{2}{n}}=P^{\frac{2}{n}} \\
\therefore \sum_{i \neq j}\left|\rho_{i}\right|\left|\rho_{j}\right| \geq n(n-1) P^{\frac{2}{n}}
\end{gathered}
$$

Now consider, $\quad\left[H E_{C}(G)\right]^{2}=\left(\sum_{i=1}^{n}\left|\rho_{i}\right|\right)^{2}$

$$
\begin{aligned}
& =\sum_{i=1}^{n}\left|\rho_{i}\right|^{2}+\sum_{i \neq j}\left|\rho_{i}\right|\left|\rho_{j}\right| \\
\therefore \quad\left[H E_{C}(G)\right]^{2} & \geq\left(|C|+2 \sum_{i<j} \frac{1}{d\left(v_{i}, v_{j}\right)^{2}}\right)+n(n-1) P^{\frac{2}{n}} \\
\text { i.e., } H E_{C}(G) & \geq \sqrt{|C|+2 \sum_{i<j} \frac{1}{d\left(v_{i}, v_{j}\right)^{2}}+n(n-1) P^{\frac{2}{n}}}
\end{aligned}
$$

Theorem 5.2. If $\rho_{1}(G)$ is the largest minimum covering Harary eigen value of $H_{C}(G)$, then $\rho_{1}(G) \geq \frac{2 \sum_{i<j} \frac{1}{d\left(v_{i}, v_{j}\right)}+|C|}{}$ value of $H_{C}(G)$, then $\rho_{1}(G)$
of minimum covering set.

Proof. Let $X$ be any nonzero vector. Then by [3], We have $\rho_{1}\left(H_{C}\right)=$ $\max _{\substack{X \neq 0 \\ \text { matrix. }}}\left\{\frac{X^{\prime} H_{C} X}{X^{\prime} X}\right\} \therefore \rho_{1}\left(H_{C}\right) \geq \frac{J^{\prime} H_{C} J}{J^{\prime} J}=\frac{2 \sum_{i<j} \frac{1}{d\left(v_{i}, v_{j}\right)}+|C|}{n}$ where $J$ is a unit

Lemma 5.1. Let $G$ be a graph of diameter 2 and $\rho_{1}(G)$ is the largest minimum covering Harary eigen value of $H_{C}(G)$, then $\rho_{1}(G) \geq \frac{2 m+n^{2}-n+2|C|}{2 n}$ where $|C|$ is the cardinality of minimum covering set. 
Proof. Let $G$ be a connected graph of diameter 2 and $d_{i}$ denotes the degree of vertex $v_{i}$. Clearly $i$-th row of $H_{C}$ consists of $d_{i}$ number of 1 's and $\left(n-d_{i}-1\right)$ number of $\frac{1}{2}$ 's. By using Rayleigh's principle, for $J=[1,1,1, \cdots, 1]$ we have

$$
\begin{array}{r}
\rho_{1}\left(H_{C}\right) \geq \frac{J^{\prime} H_{C} J}{J^{\prime} J}=\frac{\sum_{i=1}^{n}\left[d_{i} \times 1+\left(n-d_{i}-1\right) \frac{1}{2}\right]+|C|}{n} \\
=\frac{\sum_{i=1}^{n} \frac{\left(d_{i}+n-1\right)}{2}+|C|}{n}=\frac{2 m+n^{2}-n+2|C|}{2 n} .
\end{array}
$$

Similar to Koolen and Moulton's [16] upper bound for energy of a graph, upper bound for $\mathrm{HE}_{C}(G)$ is given in the following theorem.

Theorem 5.3. If $G$ is a $(m, n)$ graph with diameter 2 and

$$
\begin{aligned}
& \frac{2 m+n^{2}-n+2|C|}{2 n} \geq 1 \text { then } H E_{C}(G) \leq \frac{2 m+n^{2}-n+2|C|}{2 n} \\
& +\sqrt{(n-1)\left[\frac{4|C|+n^{2}-n+6 m}{4}-\left(\frac{2 m+n^{2}-n+2|C|}{2 n}\right)^{2}\right]} \text {. }
\end{aligned}
$$

Proof.

$$
\begin{gathered}
\text { Cauchy-Schwartz inequality is }\left[\sum_{i=2}^{n} a_{i} b_{i}\right]^{2} \leq\left(\sum_{i=2}^{n} a_{i}^{2}\right)\left(\sum_{i=2}^{n} b_{i}^{2}\right) \\
\text { Put } a_{i}=1, b_{i}=\left|\rho_{i}\right| \operatorname{then}\left(\sum_{i=2}^{n}\left|\rho_{i}\right|\right)^{2} \leq \sum_{i=2}^{n} 1 \sum_{i=2}^{n} \rho_{i}^{2}
\end{gathered}
$$

From corollary (4.2) we have,

$$
\begin{aligned}
{\left[H E_{C}(G)-\rho_{1}\right]^{2} } & \leq(n-1)\left(\frac{4|C|+n^{2}-n+6 m}{4}-\rho_{1}^{2}\right) \\
\Rightarrow H E_{C}(G) & \leq \rho_{1}+\sqrt{(n-1)\left(\frac{4|C|+n^{2}-n+6 m}{4}-\rho_{1}^{2}\right)}
\end{aligned}
$$

Let $f(x)=x+\sqrt{(n-1)\left(\frac{4|C|+n^{2}-n+6 m}{4}-x^{2}\right)}$.

For decreasing function

$$
f^{\prime}(x) \leq 0 \Rightarrow 1-\frac{x(n-1)}{\sqrt{(n-1)\left(\frac{4|C|+n^{2}-n+6 m}{4}-x^{2}\right)}} \leq 0
$$


$\Rightarrow x \geq \sqrt{\frac{4|C|+n^{2}-n+6 m}{4 n}} \therefore f(x)$ is decreasing in $\left[\sqrt{\frac{4|C|+n^{2}-n+6 m}{4 n}}, \sqrt{\frac{4|C|+n^{2}-n+6 m}{4}}\right]$. Clearly, $\sqrt{\frac{2 m+n^{2}-n+2|C|}{2 n}} \in\left[\sqrt{\frac{4|C|+n^{2}-n+6 m}{4 n}}, \sqrt{\frac{4|C|+n^{2}-n+6 m}{4}}\right]$. Since $\frac{2 m+n^{2}-n+2|C|}{2 n} \geq 1$, and by using Lemma (5.1), we have $\sqrt{\frac{2 m+n^{2}-n+2|C|}{2 n}} \leq \frac{2 m+n^{2}-n+2|C|}{2 n} \leq \rho_{1}$ $\therefore f\left(\rho_{1}\right) \leq f\left(\frac{2 m+n^{2}-n+2|C|}{2 n}\right)$ $H E_{C}(G) \leq f\left(\rho_{1}\right) \leq f\left(\frac{2 m+n^{2}-n+2|C|}{2 n}\right)$ $H E_{C}(G) \leq f\left(\frac{2 m+n^{2}-n+2|C|}{2 n}\right)$.

Therefore

$$
\begin{aligned}
& H E_{C}(G) \leq \frac{2 m+n^{2}-n+2|C|}{2 n}+ \\
& \sqrt{(n-1)\left[\frac{4|C|+n^{2}-n+6 m}{4}-\left(\frac{2 m+n^{2}-n+2|C|}{2 n}\right)^{2}\right]} \text {. }
\end{aligned}
$$

Bapat and S.pati [4] proved that if the graph energy is a rational number then it is an even integer. Similar result for minimum covering energy is given in the following theorem.

Lemma 5.2. Let $G$ be a graph with a minimum covering set $C$. If the minimum covering Harary energy $H E_{C}(G)$ is a rational number, then $H E_{C}(G)$ $\equiv|C|(\bmod 2)$.

Proof. Proof is similar to Theorem 3.7 of [1]. 


\section{References}

[1] C. Adiga, A. Bayad, I. Gutman, S. A. Srinivas, The minimum covering energy of a graph, Kragujevac J. Sci. 34 (2012) 39-56.

[2] C. Adiga and M. Smitha, On Maximum Degree Energy of a Graph Int. J. Contemp. Math. Sciences, Vol. 4, (2009), no. 8, 385 - 396

[3] R. B. Bapat, page No. 32, Graphs and Matrices, Hindustan Book Agency, (2011).

[4] R. B. Bapat, S. Pati, Energy of a graph is never an odd integer. Bull. Kerala Math. Assoc. 1, 129 - 132 (2011)

[5] S. B. Bozkurt, A. D. Güngör, B. Zhou, Note on the distance energy of graphs, MATCH Commun. Math. Comput. Chem. 64 (2010) 129-134.

[6] D. Cvetković, I. Gutman (eds.), Applications of Graph Spectra (Mathematical Institution, Belgrade, 2009)

[7] D. Cvetković, I. Gutman (eds.) Selected Topics on Applications of Graph Spectra, (Mathematical Institute Belgrade, 2011)

[8] A. Dilek Güngör, A. Sinan Çevik, On the Harary Energy and Harary Estrada Index of a Graph MATCH Commun. Math. Comput. Chem. 64 (2010) 281-296.

[9] A. Graovac, I. Gutman, N. Trinajstić, Topological Approach to the Chemistry of Conjugated Molecules (Springer, Berlin, 1977)

[10] A. D. Güngör, S. B. Bozkurt, On the distance spectral radius and distance energy of graphs, Lin. Multilin. Algebra 59 (2011) 365-370.

[11] I. Gutman, The energy of a graph. Ber. Math-Statist. Sekt. Forschungsz. Graz 103, 1-22 (1978)

[12] I. Gutman, X. Li, J. Zhang, in Graph Energy, ed. by M. Dehmer, F. Emmert - Streib. Analysis of Complex Networks. From Biology to Linguistics (Wiley - VCH, Weinheim, 2009), pp. 145 - 174.

[13] I. Gutman, in The energy of a graph: Old and New Results, ed. by A. Betten, A. Kohnert, R. Laue, A. Wassermann. Algebraic Combinatorics and Applications (Springer, Berlin, 2001), pp. 196 - 211. 
[14] I. Gutman, O. E. Polansky, Mathematical Concepts in Organic Chemistry (Springer, Berlin, 1986)

[15] Huiqing Liu, Mei Lu and Feng Tian, Some upper bounds for the energy of graphs Journal of Mathematical Chemistry, Vol. 41, No.1, (2007).

[16] J. H. Koolen, V. Moulton, Maximal energy graphs. Adv. Appl. Math. 26, $47-52(2001)$

[17] B. J. McClelland, Properties of the latent roots of a matrix: The estimation of $\pi$-electron energies. J. Chem. Phys. 54, 640 - 643 (1971)

[18] M. R. Rajesh Kanna, B. N. Dharmendra and R. Pradeep Kumar, Minimum Covering Distance Energy of a Graph. Applied Mathematical Sciences, Vol. 7, No. 111, (2013), 5525 - 5536. [ http://dx.doi.org/10.12988/ams.2013.38477]

[19] M. R. Rajesh Kanna, B. N. Dharmendra, R. Shashi and R. A. Ramyashree, Maximum degree energy of certain mesh derived networks. International Journal of Computer Applications, 78 No. 8 (2013) 38-44. [http://dx.doi.org/10.5120/13513-1289]

[20] M. R. Rajesh Kanna, B. N. Dharmendra, and G. Sridhara, Minimum dominating energy of a graph. International Journal of Pure and Applied Mathematics, 85, No. 4 (2013) 707-718. [http://dx.doi.org/10.12732/ijpam.v85i4.7]

[21] H. S. Ramane, D. S. Revankar, I. Gutman, S. B. Rao, B. D. Acharya, H. B. Walikar, Bounds for the distance energy of a graph, Kragujevac J. Math. 31 (2008) 59-68.

[22] Zhihui Cui, Bolian Liu, On Harary Matrix, Harary Index and Harary Energy, MATCH Commun. Math. Comput. Chem. 68 (2012) 815-823.

[23] B. Zhou, A. Ilić, On distance spectral radius and distance energy of graphs, MATCH Commun. Math. Comput. Chem. 64 (2010) 261-280.

[24] B. Zhou, N. Trinajstic, Maximum eigenvalues of the reciprocal distance matrix and the reverse Wiener matrix, Int. J. Quant. Chem. 108 (2008) 858-864. 
